\title{
Importancia de los estudios de anatomía patológica en COVID-19
}

\author{
Pathological anatomic studies importance in COVID-19 \\ Importância dos estudos de anatomia patológica em COVID-19
}

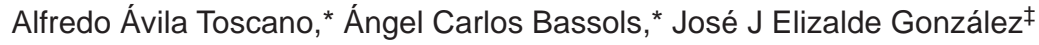

\section{RESUMEN}

El análisis histopatológico constituye el estudio rey de todos las pruebas diagnósticas en medicina. Sin embargo, las características particulares de la pandemia por COVID-19 han impuesto serias restricciones a estos análisis, por lo que la información específica al respecto es limitada. En el presente trabajo, se hace una reflexión sobre estos aspectos y se presentan algunos de los hallazgos más sobresalientes reportados en la literatura, que no se limitan a daño alveolar difuso, sino que abarcan algunas otras entidades complejas, las cuales deben analizarse con cuidado por tener importantes implicaciones terapéuticas, tanto en la estrategia de asistencia mecánica ventilatoria como en la farmacológica.

Palabras clave: COVID-19, patología, SARS-CoV-2, coronavirus, daño alveolar difuso, autopsia.

\section{ABSTRACT}

Histopathological analysis constitutes the king study of all diagnostic tests in medicine, however, the particular characteristics of the COVID-19 pandemic have imposed serious limitations on them, so that specific information in this regard is limited. A reflection is made on these aspects and some of the most outstanding findings reported in the literature are presented, that are no limited to the diffuse alveolar damage, but include some other complex entities that must be carefully analyzed, as they could have important therapeutic implications both in ventilatory and pharmacological strategies.

Keywords: COVID-19, pathology, SARS-CoV-2, coronavirus, diffuse alveolar damage, autopsy.

\section{RESUMO}

A análise histopatológica constitui o principal estudo de todos os testes de diagnóstico em medicina; no entanto, as características particulares da pandemia do COVID-19 impuseram sérias limitações a eles, de modo que as informações específicas a esse respeito são limitadas. É feita uma reflexão sobre esses aspectos e são apresentados alguns dos achados mais destacados relatados na literatura, eles não estão limitados ao dano alveolar difuso, mas incluem algumas outras entidades complexas que devem ser analisadas cuidadosamente, pois têm implicações terapêuticas importantes nas estratégias de assistência mecânica ventilatória e farmacológica.

Palavras-chave: COVID-19, patologia, SARS-CoV-2, coronavírus, dano alveolar difuso, autópsia.

... la historia sugiere que la batalla contra el SARSCoV-2 y otros coronavirus todavía está «en pañales», y que deberemos aprender lecciones no sólo para estar mejor preparados, sino también para saber sobre aspectos específicos de inmunidad de este virus, y más en general, sobre los mecanismos que apuntalan la forma grave de la infección pulmonar, incluyendo el rol de la coinfección y la desregulación inmune. ${ }^{1}$

A pesar de la progresiva disminución en la frecuencia de exámenes post mortem en todo el mundo, la au-

\footnotetext{
* Hospital Ángeles del Carmen. Guadalajara, Jalisco.

₹ Instituto Nacional de Ciencias Médicas y Nutrición «Salvador Zubirán» (INCMNSZ). CDMX.

Recepción: 25/06/2020. Aceptación: 30/06/2020.

www.medigraphic.com/medicinacritica
}

topsia sigue y seguirá siendo el estándar de oro para determinar el porqué y el cómo ocurre la muerte. La definición de la fisiopatología de la muerte no se limita sólo a consideraciones forenses, sino que también proporciona, por lo general, valiosa información clínica y de utilidad epidemiológica. Los enfoques selectivos para el diagnóstico post mortem incluyen el muestreo limitado durante la autopsia completa; son útiles, además, en el control de brotes de enfermedades, y proporcionan un conocimiento único para gestionar medidas de control adecuadas. $^{2}$

Los pacientes con COVID-19 y comorbilidad metabólica y cardiovascular son más propensos a progresar hacia formas graves de la enfermedad, e incluso hasta la muerte. ${ }^{2}$ Se ha reproducido lo ya reportado previamente al inicio de la pandemia en Wuhan: que aproximadamente $15 \%$ de los pacientes afectados por este nuevo coronavirus puede tener una presentación clínica de gravedad, y de 5 a $6 \%$ estará en condición crítica en la unidad de cuidados intensivos por datos de SIRPA (síndrome de insuficiencia respiratoria aguda) grave, inestabilidad hemodinámica, FOM (fallo orgánico múltiple) o necesidad de vigilancia estrecha. ${ }^{3-7}$

En el actual escenario, algunos autores recomiendan realizar autopsias completas en pacientes que murieron con sospecha o confirmación de infección grave por SARS-CoV-2, particularmente en presencia de diversas comorbilidades, ${ }^{2}$ pues sólo analizando una colección completa de muestras histológicas obtenidas mediante necropsia, se puede determinar la causa exacta de la muerte y reconocer patrones histológicos que ayuden eventualmente para guiar el manejo clínico.

Con la introducción de la patología digital, el campo de la patología se encuentra actualmente en un periodo de transición. Existen múltiples colecciones de diapositivas en línea gratuitas, por ejemplo, la del Colegio de Patólogos Americanos (CAP) y la de la Academia Canadiense de Patología (USCAP). Hoy por hoy, es necesario innovar el enfoque pedagógico tradicional con preguntas específicas para ayudar tanto a los alumnos a aprender como al clínico en la toma de decisiones terapéuticas. ${ }^{6}$

Desde la aparición original del SARS (síndrome respiratorio agudo grave) en China, ya hace casi 20 años, los estudios patológicos del pulmón de pacientes con 
esta enfermedad describieron similares características histológicas de daño alveolar difuso (DAD) a los cambios secuenciales relacionados con el tiempo, lo cual fue visto en DAD no asociado con SARS: edema intraalveolar e intersticial; membranas hialinas eosinofílicas que recubren la pared alveolar, algunas (incluso conteniendo neutrófilos) y escasos infiltrados mononucleares intersticiales y denudación del epitelio bronquiolar con depósitos de fibrina. En tanto, en una fase posterior (de organización), podía verse proliferación fibroblástica libre, hiperplasia de neumocitos tipo 2 , sin evidencia de inclusiones nucleares o citoplasmáticas, y células gigantes multinucleadas. ${ }^{8}$

EI SARS-CoV-2 infecta al huésped utilizando el receptor de la enzima convertidora de angiotensina 2 humana (ACE2h), que se expresa en distintos órganos de la economía (pulmones, corazón, riñones, intestino, entre otros). Los receptores ACE2h, además de las células epiteliales, también son particularmente expresados por las células endoteliales. ${ }^{9,10}$ Los ACE2h participan en múltiples procesos fisiopatológicos, incluidos los cambios patológicos de tipo lesión pulmonar aguda y tromboinflamación, ${ }^{11}$ si bien también poseen un rol en la absorción de aminoácidos neutros por el intestino (transportadores de aminoácidos), por lo que no sólo actúan como receptor abriendo la puerta al coronavirus, sino que también protegen al cuerpo de importantes cambios patológicos. ${ }^{12}$

En el subgrupo de pacientes con insuficiencia respiratoria aguda grave por COVID-19, un pequeño número de estudios post mortem ha demostrado un mayor número de células Th17 y CD8 + activadas, con células T con expresión de granzimas y perforina. Existe evidencia de activación prominente de los macrófagos en un subconjunto de pacientes con un alto nivel de ferritina e IL-6, entre otros. Esto recuerda al síndrome de activación de macrófagos, el cual frecuentemente tiene un desencadenante viral, pero esta vez, en lugar de sistémica, se asocia con una activación inmunitaria de la mucosa. ${ }^{1,3}$

Barton $L$ y colaboradores publicaron recientemente dos exámenes post mortem completos, en los cuales se realizaron procedimientos estándar en una sala de autopsias con sistema de presión negativa/sala de aislamiento, utilizando apropiado equipo de protección personal (EPP), incluidas máscaras N95, protección para los ojos y batas impermeables. El diagnóstico de COVID-19 se confirmó mediante pruebas de reacción en cadena de la polimerasa con transcriptasa inversa en tiempo real a través de hisopados post mortem. ${ }^{13}$

Las pruebas de SARS-CoV-2 se pueden realizar en la autopsia. Los hallazgos de autopsia como daño alveolar difuso y la inflamación de las vías respiratorias reflejan la verdadera patología relacionada con el virus. Otros hallazgos representan procesos superpuestos 0 no relacionados con la enfermedad en estudio (los pacientes con COVID-19 presentaron daño alveolar difuso con la formación de membranas hialinas, células mononucleares y macrófagos que se infiltran en los espacios aéreos, también con un engrosamiento difuso de la pared alveolar). La Dra. Copin y colaboradores, en Francia, describieron la presencia en biopsias post mortem de pacientes fallecidos por COVID-19 de dos entidades patológicas interesantes por ser potencialmente respondedoras a los corticosteroides: en pacientes con forma temprana de neumonía por SARS-CoV-2 (en un caso que falleció al quinto día del padecimiento), neumonía viral linfocítica, y posteriormente con formas tardías de la misma enfermedad (fallecidos alrededor de los 20 días del inicio de la enfermedad) y en pacientes con neumonía organizada fibrinoide aguda, ${ }^{14}$ que se ajustaban a las características fenotípicas funcionales descritas por Gattinoni. ${ }^{15}$ Esta última entidad se caracteriza por un depósito extenso de fibrina intraalveolar (conocido como «bolas de fibrina») más que con membranas hialinas, y que la distingue del DAD típico resultante del SIRPA clásico. Se puede añadir que el patrón obliterativo de la lesión pulmonar en neumonía grave por COVID-19 hace que nos preguntemos acerca del potencial efecto deletéreo al ofrecer niveles elevados de PEEP dentro de la estrategia de ventilación mecánica de estos pacientes: ciertamente, dicha estrategia debe llevarse a cabo de manera personalizada y dinámica.

Debemos destacar el reporte del Dr. Tian S y su grupo, en Wuhan, China, en febrero de 2020, quienes describen a dos pacientes intervenidos quirúrgicamente por patología oncológica pulmonar primaria (adenocarcinoma) y sometidos a lobectomía, los cuales estaban asintomáticos de infección respiratoria alguna, pero con diagnóstico retrospectivo de COVID-19 al momento de la operación. El examen histopatológico reportó, independientemente del tumor, una neumonía por SARSCoV-2 en fase temprana (edema, exudado proteináceo prominente, hiperplasia focal reactiva de los neumocitos con congestión vascular y grupos inflamatorios con material fibrinoide y células gigantes multinucleadas). Esta comunicación representa una de las primeras publicaciones histopatológicas de COVID-19 (Figura 1), ${ }^{16}$ separada por poco tiempo de otra realizada por Zhe Xu, la cual se publicó en línea el 17 de febrero de este año. En ella, se reportan los resultados de biopsias post mortem de un caso fatal que sirvió no sólo para identificar la causa de la muerte en particular, sino también para proporcionar nueva luz sobre la patogénesis de la neumonía asociada con COVID-19. Este trabajo ha contribuido - junto con otros de anatomía patológica- a auxiliar al clínico a formular mayores y mejores abordajes terapéuticos oportunos para pacientes críticos con las mismas características para, eventualmente, lograr reducir la mortalidad. ${ }^{17}$ 
Li y su equipo de investigación han reportado partículas virales en los bronquios y en las células epiteliales alveolares tipo 2 a través de microscopia electrónica. ${ }^{18}$

Por su parte, Lacy y otros destacan en su muy interesante trabajo qué medidas de bioseguridad se deben efectuar en el trabajo forense, para lograr así el diagnóstico post mortem de sujetos fallecidos por COVID-19. En este trabajo, se detalla ampliamente qué medidas de protección de los EPP han de cumplirse y cuáles son aquéllas que se deben efectuar posteriormente (postautopsy procedures). ${ }^{19}$ Estos autores concluyen que se debe incrementar el número de autopsias y certificar la muerte por viremia de COVID-19. Así, dependiendo de la edad, presencia o no de comorbilidades y de los criterios diagnósticos empleados, hallaron en una serie con 19 países con reporte electrónico de casos que existe una variación de 0.2 al $7.7 \%$ en el índice de mortalidad en esta enfermedad.

Chen $L$ y su grupo sugieren que los pacientes con insuficiencia cardiaca son más susceptibles a la infección cardiaca por SARS-CoV-2, y éstos podrían desarrollar más lesiones cardiacas e, incluso, la posibilidad de progresión clínica y empeoramiento. ${ }^{20}$ Se han encontrado como los hallazgos macroscópicos más significativos, a nivel cardiaco, la cardiomegalia y la dilatación del ventrículo derecho, cuya evidencia microscópica ha mostrado degeneración atípica difusa de los miocitos pero sin necrosis, lo que pudiera ser indicativo de la fase temprana de una miocarditis viral. ${ }^{21}$ Estos mismos autores han hipotetizado que los pericitos pueden estar infectados por el SARS-CoV-2, lo que produce una disfunción microvascular y de las propias células endoteliales capilares, y por tanto, se podría llevar a necrosis celular.

Los estudios de Wan Y y colaboradores (2020) mostraron varias partículas virales 2019-nCoV RBM (particularmente Gln493) que proporcionan interacciones favorables con ACE2 humano, lo que es consistente con la capacidad de SARS-CoV-2 para la infección de células humanas. ${ }^{9}$

Madjid y su equipo de investigación (2020) nos remiten a los exámenes post mortem de ocho pacientes fallecidos por SARS: cuatro de ellos tenían tromboembolia pulmonar y tres cursaron con trombosis venosa profunda. Adicionalmente, un paciente cursó con infarto subendocárdico con enfermedad coronaria oclusiva (en la presentación con SARS). Por último, otro más tenía vegetaciones valvulares gigantes de 5 a $12 \mathrm{~mm}$ que involucraban las válvulas mitral, tricúspide y aórtica, además de infarto cardiaco, afectando en riñones, el bazo y el cerebro. Sobre la misma idea, Ackermann M y colaboradores, en 10 pacientes, encontraron daño alveolar difuso, hiperplasia de neumocitos tipo 2, depósitos lineales de fibrina intraalveolar con edema intersticial moderado y depósitos de fibrina, lo que sugiere datos de organización intraalveolar temprana. 22,23
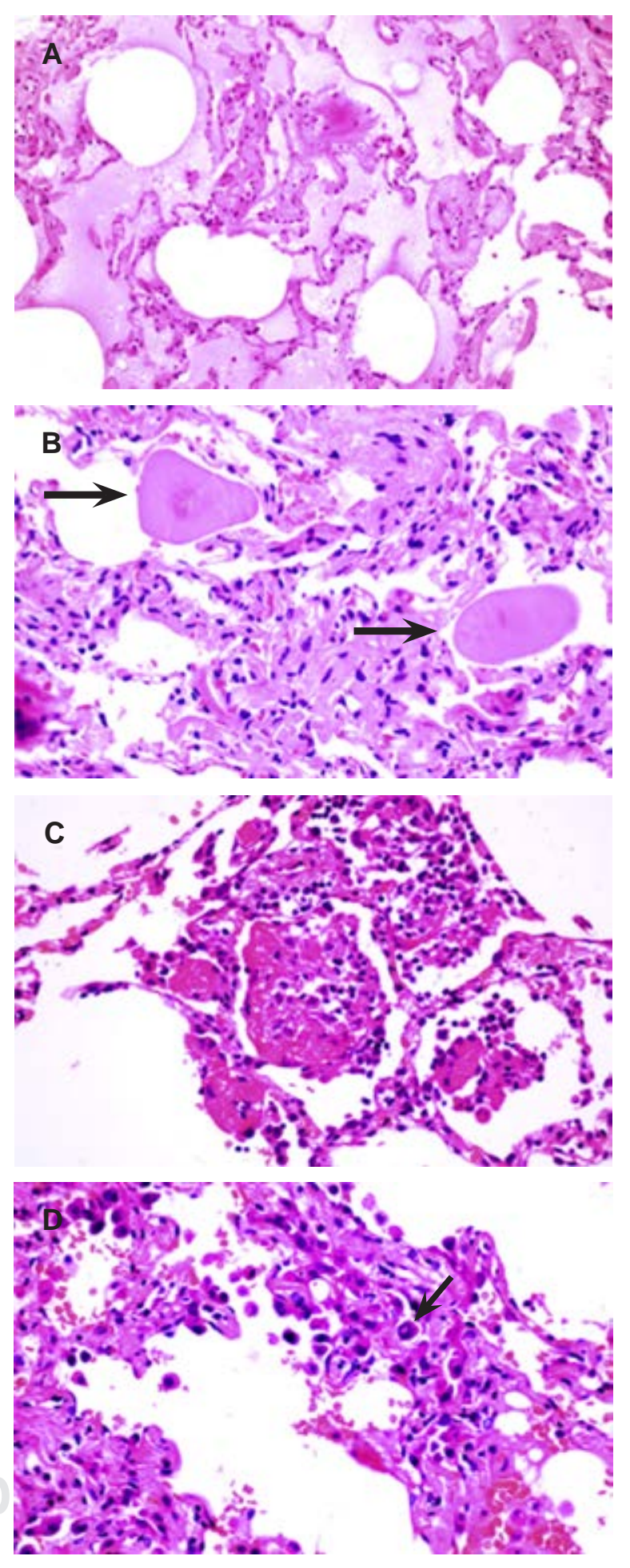

Figura 1: Cambios histológicos observados. A) Exudados proteináceos en los espacios alveolares con gránulos. B) glóbulos proteicos grandes dispersos (flechas). C) fibrina intraalveolar con organización temprana, células inflamatorias mononucleares y células gigantes multinucleadas. D) Neumocitos hiperplásicos, algunos con sospecha de inclusiones virales (flecha). Reproducido con permiso (Elsevier license number: 4866000072073). Interpretación de: Tian S, Hu W, Niu L, Liu H, Xu H, Xiao SY. Pulmonary pathology of early-phase 2019 Novel Coronavirus (COVID-19) pneumonia in two patients with lung cancer. J Thorac Oncol. 2020;15(5):700-704. 
Wichmann y colaboradores encontraron también una alta incidencia de eventos trombóticos, lo que apunta a que el virus COVID-19 induce endotelitis grave y activación anormal de la cascada de la coagulación. Por esta razón, se requieren aún de más estudios para explicar estos hallazgos, y eventualmente, lograr una posible intervención terapéutica en el futuro cercano. ${ }^{24}$

Finalmente, Merad describió microtrombos a distintos niveles en pacientes con COVID-19: pulmones, extremidades inferiores, manos, cerebro, corazón, hígado y riñones, sugiriendo que la activación de la coagulación y la coagulación intravascular son datos de una lesión orgánica en la sepsis que se asocia principalmente con citocinas inflamatorias y con la participación de la vía del factor tisular (abreviado TF; también llamado CD142 o factor de coagulación III) y que contribuye, sin duda, a la mayor gravedad de los pacientes.

Tomashesfki describió en SIRPA, desde principios de los años ochenta, cambios vasculares con tortuosidad arterial, fibrosis mural e hipertrofia de la media, así como la elevada frecuencia de fenómenos trombóticos menores y mayores. ${ }^{25}$ Más recientemente, se ha descrito la angiogénesis por intususcepción que se presenta en el COVID-19 y que se caracteriza por la separación de vasos existentes en dos a través de la formación de pilares, lo que lleva a una rápida expansión de los plexos vasculares para adaptarse a nuevos requerimientos inflamatorios y metabólicos. ${ }^{22}$ Estos hallazgos se correlacionan positivamente también con otros hallazgos de varios autores, quienes reportan la formación de trombos en las arteriolas e, incluso, infartos pulmonares y eventos tromboembólicos mayores hasta en $20 \%$ de los casos. Adicionalmente, Schaller y Claus reportaron datos inespecíficos de inflamación, los cuales son manifestados por infiltración linfocitaria; este hallazgo sugiere que existe miocarditis en fase inicial. ${ }^{21,26-29}$

Existe, sin embargo, una corriente de pensamiento distinto que sugiere que el realizar estudios post mortem en los pacientes en la epidemia actual debe estar ligado a las siguientes premisas: en primer lugar, lograr la máxima protección (reglas de bioseguridad) para todos los médicos patólogos que participen el estudio y no sólo para aquéllos que atienden pacientes con alta sospecha de infección. Ello debido a la propagación actual de la enfermedad y la tasa de falsos negativos de hisopo nasofaríngeo reportada hasta ahora. En segundo lugar, es obligatorio considerar a todos los cadáveres como potencialmente infecciosos. Finalmente, valorar los riesgos deberá normar la conducta, ya que es nuestra primera obligación salvaguardar la salud del personal de patología. ${ }^{30,31}$

Si después de la correlación clinicopatológica se considera que COVID-19 es la causa principal de muerte, entonces debe ser referido así en el formato legal (en nuestro país: Certificado de defunción). De acuerdo con datos de la Universidad Johns Hopkins, se calcula que en el mundo se han rebasado los 5,462,447 de pacientes infectados con SARS-CoV-2, siendo el reporte global de muertes en 188 países censados de un total de 344,503 , al día 25 de mayo de 2020, aunque seguramente serán muchos más, tomando en cuenta la inercia mostrada hasta ahora por la propia pandemia. ${ }^{32,33}$

A medida que evoluciona el conocimiento científico, también debemos estar abiertos al cambio y enfocarnos en que la situación actual es una oportunidad para aprender y prepararnos para potenciales futuros eventos. El Dr. Anthony S. Fauci, director del Instituto Nacional de Alergia y Enfermedades Infecciosas de los Estados Unidos de Norteamérica desde 1984, especifica el futuro de la investigación en el tema: «... el brote de COVID-19 es un claro recordatorio del desafío continuo de los patógenos infecciosos emergentes y reemergentes, y de la necesidad de una vigilancia constante, un diagnóstico rápido [...] con [amplia] investigación para comprender la biología básica de nuevos organismos». ${ }^{34}$

Se ha dicho que la muerte puede enseñarnos no sólo sobre la enfermedad, sino también puede ayudarnos con su prevención y, sobre todo, con el mismo tratamiento». El grupo de trabajo de la revista Cell nos lanza el reto: los científicos se enfrentan en este momento a un gran desafío (COVID-19), empero, estamos listos para encontrar soluciones. ${ }^{7,34,35}$

\section{BIBLIOGRAFÍA}

1. Bogaert D, Dockrell DH. 100 years of influenza research seen through the lens of COVID-19. Mucosal Immunol. 2020;13:561562. doi: org/10.1038/s41385-020-0291-9.

2. Pomara C, Volti G, Cappello F. COVID-19 deaths: are we sure it is pneumonia? Please, autopsy, autopsy, autopsy! J Clin Med. 2020;9(5):1259. doi: 10.3390/jcm9051259.

3. Huang C, Wang Y, Li X, Ren L, Zhao J, Hu Y, et al. Clinical features of patients infected with 2019 novel coronavirus in Wuhan, China. Lancet. 2020;395(10223):497-506. doi: 10. 1016/ S0140-6736(20)30183-5.

4. Wang D, Hu B, Hu C, Zhu F, Liu X, Zhang J, et al. Clinical characteristics of 138 hospitalized patients with 2019 novel coronavirus-infected pneumonia in Wuhan, China. JAMA. 2020;323(11):1061-1069. doi: 10.1001/jama.2020.1585.

5. Schaller T, Hirschbühl K, Burkhardt K, Braun G, Trepel M, Claus $\mathrm{R}$, et al. Post mortem examination of patients with COVID-19. JAMA. 2020;323(24):2518-2520. In https: jamanetwork.com/by a Mexico accese by JAMA/05/23/2020.

6. Varga Z, Flammer, Steiger A, Haberecker M, Andermatt R, et al. Endothelial cell infection and endothelitis in COVID-19. Lancet. 2020;295:1417-1418. https://doi.org/10.1016/ S01406736(20)30917-X.

7. Hanley B, Lucas S, Youd E, Swift B, Osborn M. Autopsy in suspected COVID-19 cases. J Clin Pathol. 2020;73:239-242. doi: 10.1136/jclinpath-2020-206522239.

8. Fraire AE, Libraty D, Dizon F, Olveda RM, Woda BA. Severe acute respiratory syndrome. In: Cagle PT, Allen TC. Color atlas and text of pulmonary pathology. 2a. ed. Philadelphia: Lippincott, Williams \& Wilkins; 2008, pp. 417-418.

9. Wan Y, Shang J, Graham R, Baric RS, Li F. Receptor recognition by the novel coronavirus from Wuhan: an analysis based on decade-long structural studies of SARS 
coronavirus. J Virol. 2020;94(7):e00127-20. doi: 10.1128/ JVI.00127-20.

10. Yan T, Xioa R, Lin G. Angiotensin-converting enzyme 2 in severe acute respiratory syndrome coronavirus and SARS-CoV-2: a double-edged sword? The FASEB Journal. 2020;34:6017-6026.

11. Elizalde GJJ. COVID-19 physiopathology. Med Crit. 2020; 33(3):173-175

12. Roy SF, Cecchini MJ. Implementing a structured digital-based online pathology curriculum for trainees at the time of COVID-19. J Clin Pathol. 2020;73-444. doi: 10.1136/jclinpath-2020-206682.

13. Barton M, Duval E, Stroberg E, Ghosh S, Mukhopadhyay S. COVID-19 Autopsies, Oklahoma, USA. In Am J Clin Pathol. 2020;153(6):725-733. doi: 10.1093/AJCP/AQAA062.

14. Copin MC, Parmentier E, Duburcq T, Poissy J, Mathieu D, Lille COVID-19 ICU and anatomopathology group. Time to consider histologic pattern of lung injury to treat critically ill patients with COVID-19 infection. Int Care Med. 2020. doi: orh/10-1007/ s00134-020-06057-8.

15. Gattinoni L, Chuimello D, Caironi O, Busana M, Romitti F, Brazzi L, et al. COVID-19 pneumonia: different respiratory treatment for different phenotypes? Int Care Med. 2020;46:1099-1102. doi: org/10.1007/s00134-020-06033-2.

16. Tian S, Hu W, NiuL, Xu H, Xiao SY. Pulmonary pathology of early-phase 2019 novel Coronavirus (COVID-19) pneumonia in two patients with lung cancer. $J$ of Thoracic Oncology. 2020;15(5):700-704.

17. Xu Z, Wang Y, Zhang J, Huang L, Zhang C, Liu S, et al. Pathological findings of COVID-19 associated with acute respiratory distress syndrome. Lancet Respir Med. 2020;8:420422. doi: org/10.1016/S2213-2600(20)30076-X.

18. Li H, Liu L, Zhang D, Xu J, Dai H, Tang N, et al. SARS-CoV2 and viral sepsis: observations and hypotheses. The Lancet. 2020;395:1517. doi: org/10.1016/ S0140-6736(20)30920-X.

19. Lacy J, Brooks E, Akers J, Armstrong D, Decker L, et al. COVID-19; post-mortem diagnostic and Biosafety considerations. Am J Forensic Med Pathol. 2020. doi: 10.1097/ PAF.0000000000000567.

20. Chen L, Li X, Chen M, Feng Y, Xiong Ch. The ACE2 expression in human heart indicates new potential mechanism of heart injury among patients infected with SARS-CoV-2. Cardiovasc Res. 2020;116(6):1097-1100. doi: 10.1093/cvr/cvaa078.

21. Fox SE, Akmatbekov A, Harbert JL, Li G, Brrown JQ, Vander Heide RS. Pulmonary and cardiac pathology in COVID-19: The first autopsy series from New Orleans. MedRxiv. doi: org/10.110 1/2020.04.06.20050575.

22. Ackermann M, Verleden S, Kuehnel M, Haverich A, Welte T, Laenger $\mathrm{F}$, et al. Pulmonary vascular endothelialitis, thrombosis, and angiogenesis in COVID-19. N Engl J Med. 2020;383:120218. doi: 10.1056/NEJMoa2015432.

23. Madjid M, Safavi-Naeini P, Solomon SD, Vardeny O. Potential effects of coronaviruses on the cardiovascular system a review. JAMA Cardiol. 2020. doi: 10.1001/jamacardio.2020.1286.

24. Wichmann D, Sperhake JP, Lütgehetmann M, Steurer S, Edler C, Heinemann A, et al. Autopsy findings and venous thromboembolism in patients with COVID-19: a prospective cohort study. Ann Int Med. 2020; doi: 10.7326/M20-2003.
25. Tomashefski JF Jr, Davies P, Boggis C, Greene R, Zapol WM, Reid LM, et al. The pulmonary vascular lesions of the ARDS. Am J Pathol. 1983;112:112-126.

26. McGonagle D, O'Donnell J, Sharif K, Emery P, Bridgewood Ch. Why the immune mechanisms of Pulmonary Intravascular Coagulopathy in COVID-19 pneumonia are distinct from macrophage activation syndrome with disseminated intravascular coagulation. The Lancet. 2020;2(7):E437-E445. Disponible en: www.reserchgate.net/publication/doi: 10.13140/ RG.2.2.19782.83521

27. Buja M, Wolf D, Zhao B, Akkanti B, McDonald M, Lelenwa L, et al. Emerging spectrum of cardiopulmonary pathology of the coronavirus disease 2019 (COVID-19): report of three autopsies from Houston, Texas and review of autopsy findings from other united states cities. Cardiovasc Pathol. 2020;48:107233. doi: org/10.1016/j.carpath.2020.107233.

28. Carsana L, Sonzoguia A, Nasr A, Rossi RS, Zerbi P, et al. Pulmonary post-mortem findings in the large series of COVID 19 cases form North Italy: a two-centre descriptive study. medRvix. 2020. doi: org/10.1101/2020.04.19.20054262.

29. Merad M, Martin J. Pathological inflammation in patients with COVID-19: a key role for monocytes and macrophages. Nat Rev Immunol. 2020;20:355-362. doi: org/10.1038/s41577-0200331-4.

30. Barbarareschi M, Facchetti F, Fraggetta F, et al. What are the priorities of pathologists' activities during COVID-19 emergency? Pathologica Epub. 2020;112(2). https://doi.org/10.32074/1591951X-15-20.

31. Parraguirre MS, De Anda MGJ, Mantilla A, Uribe NO. Guías de manejo en el laboratorio de Anatomía Patológica de cadáveres y material biológico de pacientes con diagnóstico o sospecha de COVID-19. Patología Rev Latinoam. 2020;58:1-9. doi: org/10.24245/patrl.v58id.4253.

32. Rao CH. Medical certification of cause of death for COVID-19. In www.who.int/bulletin/volumes/98/5/20-257600.

33. COVID-19 dashboard by the Center for system science and engineering (CSSE) at John Hopkins University (JHU) /25/05/2020.

34. Fauci A, Lane HC, Redfield R. On progress made during the past four decades of the HIV/AIDS pandemic and ongoing efforts to end this threat. Supplement to the N Eng J Med 2020; 383:1-4

35. The Cell Editorial Team. COVID-19: navigating uncertainties together. Cell. 2020;181(2):209-210. doi: org/10.1016/j. cell.2020.03.04.

Conflicto de intereses: Los autores declaramos no tener conflicto de intereses.

Correspondencia:

José J Elizalde González

E-mail: jjeg@unam.mx

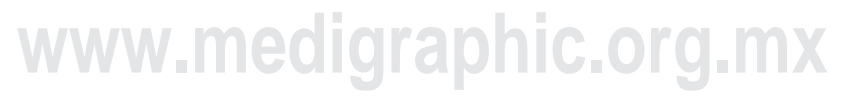

